\title{
Design of the PROSPECT Experiment
}

\author{
Pranava Teja Surukuchi* for the PROSPECT Collaboration \\ Illinois Institute of Technology \\ E-mail: psurukuc@hawk.iit.edu
}

PROSPECT is a multi-phased reactor antineutrino experiment with primary goals of making a precise measurement of the ${ }^{235} \mathrm{U}$ reactor antineutrino spectrum and performing a search for sterile neutrinos. The detectors will be deployed at short baselines of $\sim 7-19 \mathrm{~m}$ from the High Flux Isotope Reactor at Oak Ridge National Laboratory. In addition to being optimally located to search for oscillations arising from $\Delta \mathrm{m}^{2} \sim 1 \mathrm{eV}^{2}$ scale sterile neutrinos, the short distance of the detector from the reactor provides a large $\bar{v}_{e}$ flux from the reactor core. However, the close proximity also poses challenges such as, constraints on space, lack of over-burden, and backgrounds from the reactor. Therefore, the detector must be designed to have excellent background rejection capabilities, along with good position and energy resolutions, to meet the physics goals of the experiment. The baseline PROSPECT detector design consists of a ${ }^{6} \mathrm{Li}$-loaded liquid scintillator segmented detector. In this poster, we give a detailed description of the experimental strategy of PROSPECT, the PROSPECT detector design, and supporting R\&D activities.

38th International Conference on High Energy Physics

3-10 August 2016

Chicago, USA

${ }^{*}$ Speaker. 


\section{Introduction}

Reactors have played an important role in the history of neutrino physics. Recent reactor antineutrino experiments $[1,2,3]$ have precisely measured the smallest mixing angle, $\theta_{13}$, which has helped to establish a comprehensive picture of mixing in the neutrino sector. However, these experiments have also reported an antineutrino flux that differs by $\sim 6 \%$ compared to the theoretical predictions [4, 5]. Sterile neutrinos on the order of $\sim 1 \mathrm{eV}^{2}$ that can oscillate to standard neutrinos have been invoked [6] to explain this deficit. In addition to the flux deficit, these experiments have also reported deviations from the predicted antineutrino spectrum in the 4-7 MeV region. Errors in reactor antineutrino production models arising from an incomplete understanding of nuclear physics could explain these deviations. PROSPECT is an experiment designed to resolve these anomalies. The primary goals of PROSPECT are an unambiguous oscillation measurement to search for the existence of $\mathrm{eV}$-scale sterile neutrinos via electron-antineutrino disappearance and a precise measurement of the ${ }^{235} \mathrm{U}$ antineutrino spectrum to test the reactor antineutrino spectrum predictions.

\section{Antineutrino Source}

The antineutrino source for the PROSPECT experiment is the High Flux Isotope Reactor (HFIR) located at Oak Ridge National Laboratory. HFIR is a compact Highly Enriched Uranium (HEU) research reactor operating at $85 \mathrm{MW}$ for approximately $47 \%$ of the year. The small diameter of the HFIR is inherently advantageous because it avoids washing out the oscillation effect for meter-long oscillations. The use of HEU fuel, which is principally made of ${ }^{235} \mathrm{U}$, in conjunction with a short reactor-on cycle, means that ${ }^{235} \mathrm{U}$ contributes to almost all of the fissions in the HFIR core throughout the cycle. As a consequence, most of the antineutrinos emitted by HFIR originate from the daughters of ${ }^{235} \mathrm{U}$ which allows for an accurate assessment of the reactor flux models.

The PROSPECT collaboration has performed a comprehensive background assessment at the HFIR reactor site [8]. This information, in conjunction with the PROSPECT prototypes deployed at the reactor site, has played a crucial role in the design and development of the PROSPECT detector and the passive shielding.

\section{Antineutrino Detector}

PROSPECT is a phased experiment with a movable Phase-I Antineutrino Detector (AD-I) covering baselines of 7-12 m, while the Phase-II detector extends this baseline to $19 \mathrm{~m}$. The oscillation search in PROSPECT experiment will be performed by comparing the detected antineutrino spectrum as the function of baseline. The movable nature of $\mathrm{AD}-\mathrm{I}$, in addition to providing a wide range of baseline coverage, also gives a concrete way to reduce baseline-dependent systematic biases.

\subsection{Liquid Scintillator}

The target for the PROSPECT experiment is $\sim 30001$ of ${ }^{6} \mathrm{Li}$-loaded EJ-309 liquid scintillator (LiLS) with Pulse Shape Discriminating (PSD) capabilities. Antineutrinos $\left(\bar{v}_{e}\right)$ from the reactor are detected via inverse beta decay (IBD) interaction $\left(\bar{v}_{e}+p \rightarrow n+e^{+}\right)$. The outgoing positron 


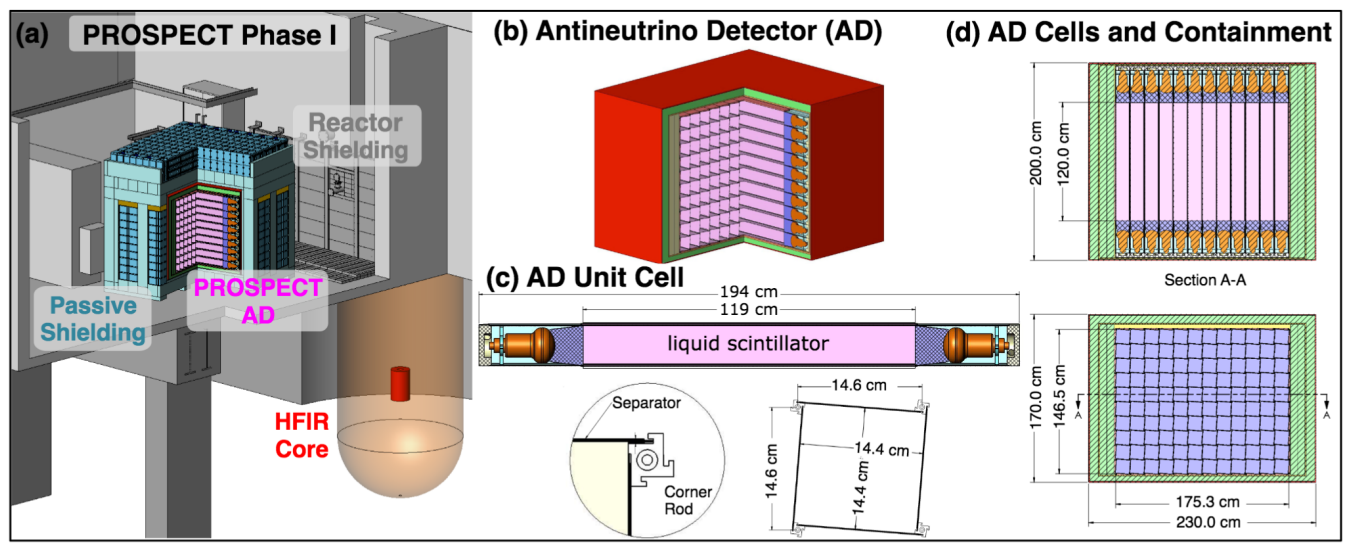

Figure 1: (a) Schematic of the AD-I detector at the HFIR reactor site. (b) Cutaway of AD-I. (c) Schematic of a unit segment and the constituent components of a segment. (d) Section views of the detector segments and containment.

carries most of the neutrino energy, it annihilates with an electron and produces a prompt signal. The neutron thermalizes and captures on ${ }^{6} \mathrm{Li}$ or hydrogen to produce a delayed signal. The capture on ${ }^{6} \mathrm{Li}$ produces a spatially compact signal with a characteristic delay time of $\sim 40 \mu \mathrm{s}$ and a characteristic quenched energy of $\sim 0.6 \mathrm{MeV}$. The spatial and temporal coincidence, in addition to the characteristic energy deposition, reduces the uncorrelated backgrounds. Moreover, the PSD capability of the LiLS can be used to veto the neutron and $\gamma$ correlated backgrounds by placing PSD cuts on both prompt and delayed signals.

\subsection{Segmentation}

PROSPECT AD-I is designed as a single volume of LiLS that is optically isolated into segments by highly-reflective low-mass separator panels. Each segment has two PMTs one at each end. Segmentation of AD-I provides a way to compare the measured reactor spectrum between segments, eliminating the reliance on reactor models. It also aids in background rejection by providing topology information as well as a convenient fiducial boundary enabling the outer layer of segments to act as a $\gamma$ catcher.

The segmented nature of the AD-I design provides an inherent position resolution in the directions corresponding to the width of a segment. Furthermore, the relative difference between timing and charge of the signal observed at each PMT can be used to reconstruct the position of an event along the length of a segment. As detailed in [8], double-ended readout also provides excellent and uniform light collection efficiency as well as superior PSD capabilities. Figure 1 shows schematics of AD-I in the HFIR facility along with the detailed views of the segments, the separator panels, and their dimensions.

\subsection{Detector Calibration}

The optical separators are supported by custom-designed 3D-printed Polylactic Acid (PLA) pieces. As shown in Figure 1, the PLA pieces are hollow providing convenient locations for insitu deployment of optical and source calibrations. The light transmission, PMT gain, and timing information will be calibrated and monitored using a pulsed laser source via optical fibers. The 
detection efficiency, absolute and relative energy scales, scintillator non-linearity, and PSD will be calibrated periodically using retractable encapsulated $\gamma$ and neutron sources. In addition, the cosmogenic backgrounds, in conjunction with calibration sources, can be used to monitor the detector response.

\section{Shielding Design}

Detailed background studies at the HFIR reactor site have shown spatial variations in the reactor related backgrounds [8]. PROSPECT prototype detectors, along with various shielding tests, show that the reactor related backgrounds can be significantly reduced by targeted passive shielding. Fixed lead walls will be used to suppress reactor-related gamma backgrounds and a movable multi-layer shielding package will reduce the reactor-related neutron and cosmogenic backgrounds. These passive shielding measures in conjunction with topology, timing, PSD, and fiducialization analysis cuts are predicted to reduce the backgrounds and achieve a signal-to-background of approximately $3: 1$, which is sufficient to reach the PROSPECT physics goals.

\section{Acknowledgments}

This material is based upon work supported by the U.S. Department of Energy Office of Science. Additional support for this work is provided by Yale University, the Illinois Institute of Technology, the National Institute of Standards and Technology, and the Lawrence Livermore National Laboratory LDRD program. We gratefully acknowledge the support and hospitality of the High Flux Isotope Reactor at Oak Ridge National Laboratory, managed by UT-Battelle for the U.S.Department of Energy.

\section{References}

[1] [Daya Bay Collaboration], Measurement of the Reactor Antineutrino Flux and Spectrum at Daya Bay,' Phys. Rev. Lett. 116(6) 061801 [hep-ex/1508.04233].

[2] Double Chooz Collaboration, Improved measurements of the neutrino mixing angle $\theta_{13}$ with the Double Chooz detector, JHEP 1410, 086 [JHEP 1502, 074] [hep-ex/1406.7763].

[3] RENO Collaboration, Observation of Energy and Baseline Dependent Reactor Antineutrino Disappearance in the RENO Experiment, Phys. Rev. Lett. 116(21)211801 [hep-ex/1511.05849].

[4] P. Huber, On the determination of anti-neutrino spectra from nuclear reactors, Phys. Rev. C 84(2)024617 [hep-ph/1106.0687v4]

[5] T. Mueller et al., Improved predictions of reactor antineutrino spectra, Phys. Rev. C 83(5) 054615[hep-ex/1101.2663v3]

[6] K. N. Abazajian et al., Light Sterile Neutrinos: A White Paper, [hep-ph/1204.5379].

[7] PROSPECT Collaboration, The PROSPECT Physics Program, J. Phys. G 43,(11) 113001 [physics.ins-det/1512.02202].

[8] PROSPECT Collaboration, Background Radiation Measurements at High Power Research Reactors, Nucl. Instrum. Meth. A 806, (401) 2016 [physics.ins-det/1506.03547]. 